\title{
Analysis of Traffic Conflicts in a Mixed-Airspace Evaluation of Airborne Separation Assurance
}

\author{
Timothy A. Lewis* \\ NASA Langley Research Center, Hampton, VA 23681, USA
}

\begin{abstract}
A pair of human-in-the-loop simulation evaluations of a distributed air/ground separation assurance system have been conducted to investigate the function allocation between humans and automation systems as well as ground-based and airborne agents in the Next Generation Air Transportation System and beyond. This paper focuses on an analysis of certain critical conflicts observed between self-separating aircraft and ground-managed traffic in the same airspace. The principal cause of each conflict is identified and potential mitigations are discussed, such as: the sharing of trajectory intent information between the ground and the air; more cautious trajectory planning by the self-separating aircraft; and more equitable rules-of-the-road between the self-separating aircraft and ground-managed aircraft. This analysis will inform the ongoing design of an airborne separation assurance automation tool.
\end{abstract}

\section{Introduction}

Separation assurance is a critical service provided by air traffic controllers to aircraft operating under Instrument Flight Rules (IFR). How and by whom this function will be performed in the future is a pivotal concern in the developing Next Generation Air Transportation System (NextGen). The allocation of separation assurance functions between humans and automated systems, between agents on the ground and in the air, and between centralized and distributed control paradigms has great implications for the evolution of air traffic management and the future of air transportation. This question is among those being explored by the Concepts and Technology Development Project under the NASA Airspace Systems Program.

To investigate, NASA has developed operational concepts and enabling technologies for en route separation assurance and trajectory management that change the allocation of functions from the state of the practice today. In the concept presented here, en route controllers will use automation tools of increasing sophistication to manage greater numbers of IFR aircraft, thus increasing airspace capacity and reducing the costs of the ground-based operation. In the air, a new self-separation operation will allow aircraft to fly autonomously and independently from ground-based control, providing greater operational flexibility for those operators that choose to equip. Together, this air/ground concept for separation assurance represents a promising allocation of functions for NextGen and beyond.

This concept has recently been evaluated in a pair of human-in-the-loop simulation experiments, focusing on the interaction between IFR traffic managed by an advanced ground-based air traffic control system, and self-separating aircraft following what has been dubbed the Autonomous Flight Rules (AFR) concept of operations. One experiment focused on the perspective of the IFR controller, and the other on the AFR pilot. This paper presents an analysis of the AFR operation and some practical challenges arising at the confluence of AFR and IFR traffic in the same airspace. In particular, the principal causes of certain critical conflicts observed between AFR and IFR aircraft are identified, and potential mitigations are discussed. The results of this analysis have implications for design of the conflict detection and resolution algorithms in the air, rules-of-the-road between ground-managed and self-separating aircraft in mixed airspace, and the information-sharing architecture of NextGen and beyond.

\footnotetext{
*Aerospace Engineer, Crew Systems and Aviation Operations Branch, M/S 152, AIAA Member.
} 


\section{Background}

\section{A. Concept of Operations}

The concept presented here consists of essentially two distinct separation assurance systems: i) a ground-based system responsible for providing separation to conventional IFR traffic, and ii) a distributed airborne system in which equipped aircraft self-separate from other traffic according to AFR rules. These systems have been designed in a compatible way so that the two operations may coexist in the same airspace; i.e., a mixture of both operations, reflecting a variety of equipage levels and different uses of the airspace.

\section{Ground-Based Separation}

The ground-based concept represents an evolution of the air traffic control (ATC) system and IFR operations as they exist today. In this concept, controllers are assisted by a set of decision support tools with which to manage an increased number of aircraft equipped to receive data link clearances. These features serve to multiply the number of aircraft that can be managed by a single human controller. However, the relationship between the controller and the IFR pilot remains unchanged from today: the controller is responsible for ensuring separation while accommodating the needs of the airspace user, and the IFR pilot is responsible for following ATC clearances and instructions.

\section{Airborne Separation}

In parallel to ground-based separation is the airborne concept for self-separation under AFR. This operation is enabled by the availability of Automatic Dependent Surveillance-Broadcast (ADS-B) as well as the development of onboard decision support tools to assist the pilot in performing separation functions. With AFR, an onboard tool continuously scans the ADS-B surveillance information broadcast from nearby traffic and alerts the pilot if any conflicts are detected. In that event, the tool recommends a strategic route modification or a tactical vector to be flown in order to resolve the conflict.

To fly AFR, i) the operator must equip with ADS-B IN and other avionics to support the onboard automation tool, and ii) the pilot must take on the responsibility for self-separation during the en route phase of flight. In return, AFR is envisioned to allow the operator to better achieve its business-optimal trajectory through greater control over routes, altitudes, and speeds while being subject to fewer ground-based traffic flow constraints.

\section{Mixed Operations}

In mixed operations, IFR and AFR aircraft coexist in the same airspace at the same flight levels. The pair of experiments presented here considered one particular division of separation responsibility. In this division, the controller remains responsible for IFR traffic, resolving conflicts arising among IFR aircraft. At the same time, the AFR aircraft self-separate and resolve conflicts with other AFR aircraft as well as with IFR traffic. Thus, AFR aircraft relieve the controller of the burden of responsibility for handling AFR/IFR conflicts. This design decision allows AFR aircraft to operate independently of the ground without increasing the workload of the IFR controller or substantially changing the design of the air traffic control system as it is today. This decision is being considered as a transition step for the initial implementation of airborne separation; NextGen in the far-term might see a more equitable distribution of conflict resolution responsibility between the air and the ground. A thorough description of the AFR concept and AFR/IFR mixed operations can be found in Refs. 1 and 2.

\section{B. Human-in-the-Loop Simulation Experiment}

The mixed-operations concept was evaluated in a pair of jointly conducted human-in-the-loop simulation experiments completed in late 2012. The first experiment, conducted in the Airspace Operations Laboratory at NASA Ames Research Center, focused on the effect of the AFR aircraft on the ability of the controller to manage the IFR traffic. The second experiment, conducted in the Air Traffic Operations Laboratory at NASA Langley Research Center, focused on the ability of the AFR pilot to resolve all AFR/IFR conflicts arising in mixed airspace. A complete discussion of both of these experiments and their results can be found in Ref. 3. This paper focuses on the "exploratory" component of the airborne-focused experiment conducted at Langley.

In the exploratory component, each week featured six AFR crews consisting of two airline pilots each. Each crew flew six scenarios of 30 minutes (min) in length through Cleveland Air Route Traffic Control Center (ARTCC) airspace populated by simulated IFR traffic managed by three air traffic controllers. Two weeks of this data were collected. 
These runs were intended to evaluate the mixed operation from the perspective of the AFR pilot under typical en route conditions without any scripted anomalies or off-nominal scenarios. All flying was conducted in cruise before top of descent.

Further, these runs examined the effect of trajectory intent information sharing from the IFR aircraft to the AFR aircraft. In half of the runs, the IFR aircraft broadcast only the ADS-B state vector (position and velocity) in accordance with the FAA's 2020 minimum equipage mandate. ${ }^{4}$ In the other half, the IFR aircraft also broadcast up to four ADS-B trajectory change points, representing a future NextGen condition with greater trajectory information availability for flight deck users. This paper focuses on the state-only, intent-poor runs of the first half, which represent a more challenging condition for the AFR aircraft.

The experiments employed the Aircraft Simulation for Traffic Operations Research (ASTOR) for AFR aircraft, which is a desktop computer simulation of a commercial transport with a modern glass cockpit based on the Boeing 777. The ASTOR simulators were connected to the Multi-Aircraft Control System (MACS), simulating the Display System Replacement (DSR) interface with advanced controller tools at Cleveland Center used to control the IFR traffic.

\section{Analysis of Conflicts}

\section{A. Preliminaries}

This analysis uses the 5 nautical miles (nmi) lateral and 1000 feet ( $\mathrm{ft}$ ) vertical separation standards commonly adopted for en route flight. Aircraft closer than this standard are said to be in loss of separation (LOS). No losses of separation were observed in all of the exploratory runs in the Langley experiment.

Two aircraft are said to be in conflict when a comparison of their predicted trajectories reveals a future LOS within a certain time horizon; in this experiment, 10 min. Onboard each AFR aircraft, the Autonomous Operations Planner (AOP) software tool continuously scans the ADS-B broadcasts for conflicts with surrounding traffic. Upon detection of a conflict, AOP alerts the AFR crew and provides a set of appropriate conflict resolution maneuvers for the pilot to execute. The reader is directed to Ref. 5 for a detailed description of AOP.

Ideally, each conflict would be detected at the time horizon of $10 \mathrm{~min}$. However, due to the error and uncertainty involved in trajectory prediction, and the dynamic nature of maneuvering air traffic, conflicts may arise and be detected with less than the maximum warning time. In the worst cases, an AFR aircraft may have under a minute to react to an IFR conflict.

The amount of warning time is generally related to the "difficulty" of the required conflict resolution from the AFR pilot's perspective. A conflict with 5 to $10 \mathrm{~min}$ of warning time can be solved strategically using a modified route via the flight management system (FMS). Under $5 \mathrm{~min}$, a more tactical resolution is required, given to the pilot as a vector or altitude change to fly directly using the mode control panel. Conflicts detected with less than 5 min to go are known as pop-up conflicts. As time grows short and the aircraft progress closer to LOS, larger maneuvers are required to avoid the conflict and the workload increases for the AFR pilot.

As discussed before, the AFR aircraft in the experiment were responsible for resolving all conflicts arising with IFR traffic. In order to provide extra "breathing room," AOP was configured to apply a lateral separation standard of $8 \mathrm{nmi}$ rather than $5 \mathrm{nmi}$ to IFR aircraft. The extra distance is known as the IFR buffer. For the remainder of the paper, conflict detections and reported values for warning time are derived from AOP's trajectory predictions using a lateral separation standard of $8 \mathrm{nmi}$.

For the AFR operation to be acceptable and beneficial in practice, pop-up conflicts with a very short amount of warning time must be a rare event. This is a crucial concern for the design of the separation assurance system on the flight deck, the controller procedures and automation tools, as well as the information sharing architecture of NextGen.

The following analysis is focused on the AFR/IFR pop-up conflicts observed in the experiment that meet the following conditions:

1. The AFR aircraft was in lateral navigation (LNAV) and vertical navigation (VNAV) autoflight system modes at the moment of conflict detection. In this state, the autoflight system is "fully coupled" with the FMS, and it is considered to be the nominal mode for AFR flight.

2. The amount of warning time at conflict detection was less than or equal to $5 \mathrm{~min}$. During simulation debrief sessions, pilot and controller participants suggested this as a minimum amount of time for a conflict to be considered normal and routine.

While there were many other conflicts observed during the experiment, it is warranted to give extra attention to those meeting these conditions. These conflicts tend to be "initiator" events, marking the transition between routine 
AFR flight and a period of significantly increased workload for the AFR pilot. The following analysis is concerned with the common causes of these conflicts and potential mitigations thereof.

\section{B. Conflict Causes and Mitigations}

The remainder of the paper will use the terminology of ownship to denote the AFR aircraft and intruder for the IFR aircraft in a given conflict encounter.

Table 1 shows a tally of the vertical conditions of the ownship and intruder for all conflicts at the moment of initial detection. In 33 out of the 35 conflicts, either the ownship or the intruder was involved with some sort of vertical maneuver; in only two of the cases were both aircraft level and co-altitude. Twenty-six of the conflicts occurred when the ownship was level, and 14 of those involved an intruder leveling off at top-of-climb. It is clear that there is an influence between altitude changes by either aircraft and the occurrence of pop-up conflicts, more so than lateral maneuvering alone.

Table 1. Tally of ownship and intruder vertical conditions at initial conflict detection.

\begin{tabular}{cc|ccccccc|c}
\hline \hline & \multicolumn{7}{|c|}{ Intruder } & \\
& & LVL & BOC & CLB & TOC & TOD & DES & BOD & \\
\hline \multirow{5}{*}{ Ownship } & LVL & 2 & 4 & 2 & 14 & 4 & 0 & 0 & 26 \\
& BOC & 2 & 0 & 1 & 0 & 0 & 0 & 0 & 3 \\
CLB & 2 & 0 & 0 & 0 & 0 & 0 & 0 & 2 \\
& TOC & 3 & 0 & 0 & 0 & 0 & 0 & 0 & 3 \\
& TOD & 0 & 0 & 0 & 0 & 0 & 0 & 1 & 1 \\
& DES & 0 & 0 & 0 & 0 & 0 & 0 & 0 & 0 \\
BOD & 0 & 0 & 0 & 0 & 0 & 0 & 0 & 0 \\
\hline \hline & 9 & 4 & 3 & 14 & 4 & 0 & 1 & 35 \\
\hline
\end{tabular}

LVL Level flight

BOC Bottom of climb

CLB Climbing flight

TOC Top of climb

TOD Top of descent

DES Descending flight

BOD Bottom of descent

A full list of conflicts is given in Table 2 in the Appendix. The following subsections describe a set of common conflict causes identified by a careful examination of the recorded data from each ownship flight.

\section{Cause 1: Intruder is climbing or descending and levels out at the ownship's altitude.}

The intruder is climbing from below or descending from above the ownship, which is flying level. AOP does not detect a conflict with the intruder's predicted climb or descent trajectory; there is no conflict with the intruder if it continues to climb or descend at its current vertical speed. However, at the moment that the intruder levels out at the ownship's altitude, a conflict is detected on the new, level trajectory. (4 of 35 cases)

Example: (Conflict 1) The ownship is level at FL370 a and the intruder is climbing from below on a crossing path from the right. Intruder levels out at FL370 and causes a pop-up conflict with the ownship with 1:32 min of warning. The ownship makes a left turn to resolve the conflict, following AOP's guidance.

The trajectories for the ownship and intruder are shown in Fig. 1. In this figure and subsequent others, the following conventions are used:

- The ownship trajectory is shown in blue and the intruder trajectory in red. The conflict duration on both plots is highlighted in amber $(\square)$.

- In the lateral plot, the trajectories are shown in a local north $(\mathrm{N})$ and east $(\mathrm{E})$ coordinate system with distances in nautical miles. Each aircraft's initial position and heading is marked by a chevron. The as-flown trajectory for each aircraft is shown in solid (-), and the trajectory prediction of each aircraft made by AOP on the ownship at the time of initial conflict detection is shown in dashed (-- ).

- The vertical plot shows the flight level (FL) versus time for each aircraft. The time shown is the number of seconds (s) since the start of the simulation run. The time span shown here corresponds to the as-flown trajectory segment shown in the lateral plot. For both aircraft, the as-flown trajectory is shown in solid (-). The last conflict-free trajectory prediction that AOP made before the conflict detection is shown in dashed (- - -).

${ }^{\mathrm{a}} \mathrm{FL}$ denotes the flight level altitude convention based on barometric pressure, in hundreds of feet. FL370 denotes a flight level of 37,000 ft. 
The trajectory prediction at the initial conflict detection (i.e., the trajectory upon which the detection is based) is shown in dash-dot (-- - - - The dash-dot trajectory is not visible in Fig. 1 because it is concident with the as-flown trajectory in solid.
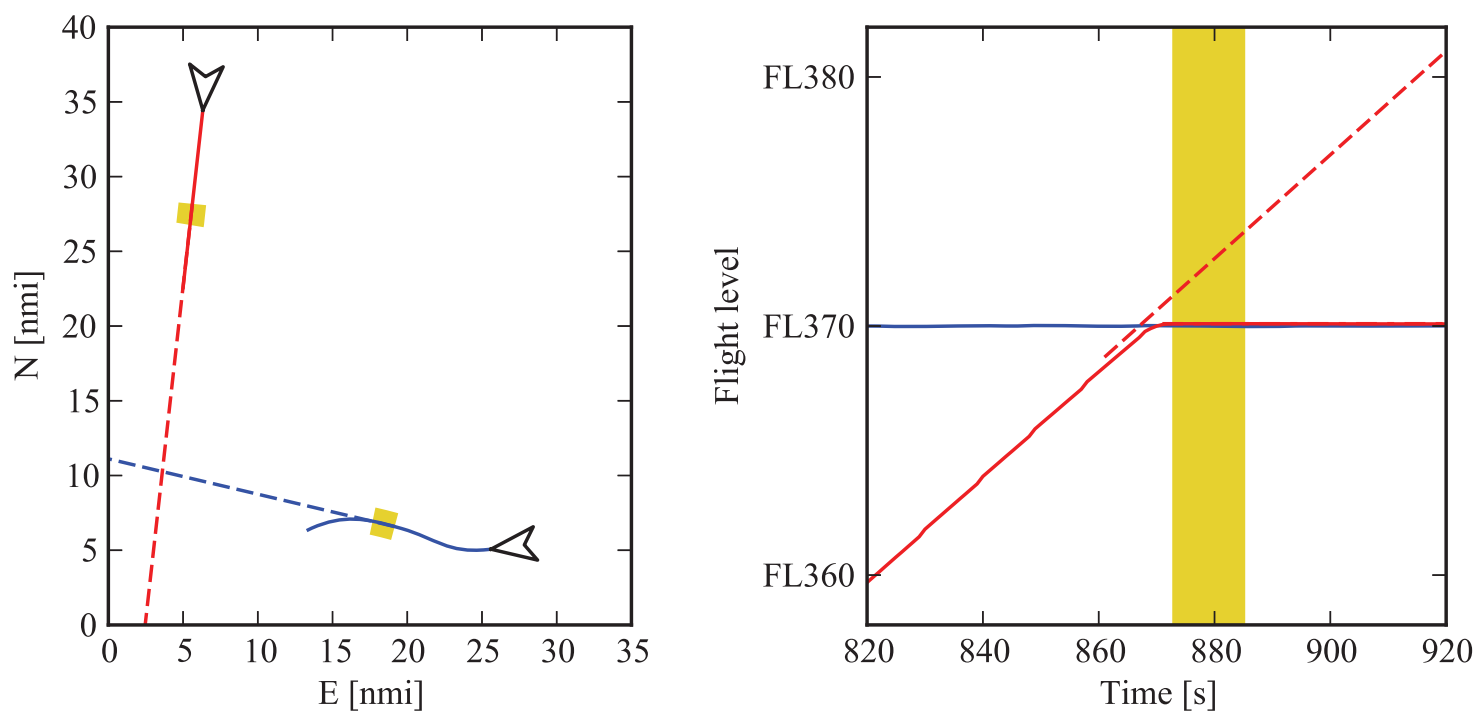

Figure 1. Conflict 1 trajectories. Left: lateral, right: vertical.

This encounter can be considered a missed detection. AOP did not detect this conflict because it was not aware of the altitude to which the intruder was cleared to climb. Several mitigations for this case are possible:

- The ownship must be provided with the intruder's target altitude. This could be obtained by a number of ways, for example: the ADS-B target state report, whereby the intruder broadcasts the current value in the altitude window of its mode control panel; or by a data link of the intruder's clearance altitude from the ground-based controller automation system. With this target altitude, AOP can predict when the intruder will level out and provide the pilot with more warning time.

- AOP must be more conservative when in the proximity of such an intruder, assuming that the intruder can level out at any altitude. This approach could provide the pilot with more warning time at the cost of more false alarms.

- The controller or ground-based automation must not be permitted to level out the intruder at the ownship's altitude and create such a conflict. In the example here, the controller might hold the intruder at a lower altitude until it passes by the ownship, thereafter clearing it to FL370.

It should be noted that in the real world a controller would not be comfortable with allowing such a hazard to unfold without intervention. However, in the experiment, controllers were instructed that the AFR aircraft were responsible for and capable of providing all separation from IFR traffic. Thus, they allowed many of these conflicts to unfold for the sake of testing the AOP tool.

Cause 2: Intruder is climbing but will level out below the ownship.

The intruder is flying level below the ownship, and the pair are conflict free. Then, the intruder begins a climb and AOP detects a conflict on the intruder's new trajectory. However, the intruder is cleared to a lower altitude than the ownship's current altitude, and will level out harmlessly below. (4 of 35 cases)

Example: (Conflict 4) The ownship is level at FL390 and the intruder is level at FL370 on a crossing track from the left. The intruder begins a climb and AOP detects a conflict with 1:57 min of warning. The pilot initiates an AOPguided right turn to resolve the conflict. However, the intruder levels out at the cleared altitude of FL380, passing below the ownship. This conflict is shown in Fig. 2. 

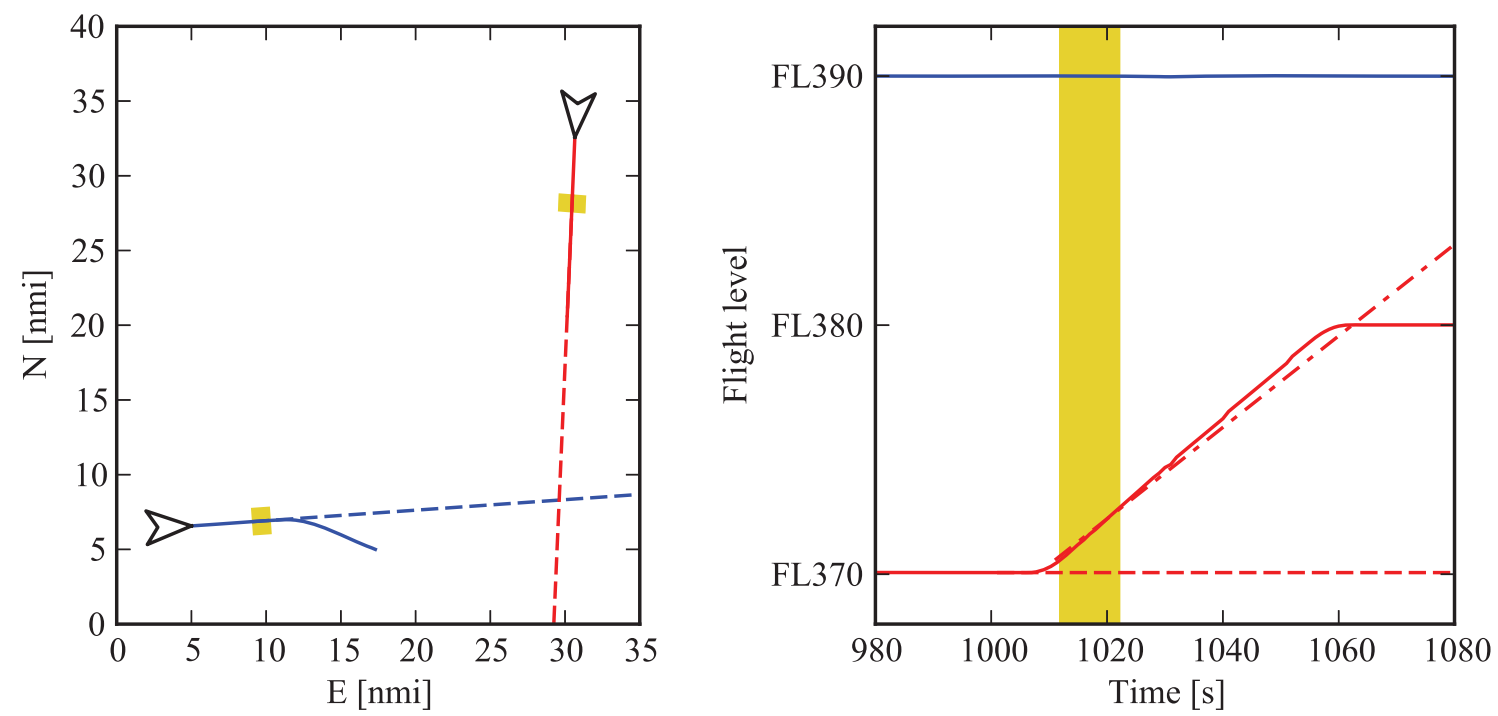

Figure 2. Conflict 4 trajectories. Left: lateral, right: vertical.

This situation can be considered a false alarm. AOP did not know the intruder's intended altitude and assumed it would continue climbing at the instantaneous vertical speed and enter into LOS with the ownship. In reality, the intruder presented little risk to the ownship and the resolution maneuver was ultimately unnecessary.

Potential mitigations for this cause:

- As discussed in the previous example, AOP can avoid this situation if it is provided with the intruder's target altitude via broadcast or data link.

- Suppose AOP knows with high certainty that the controller or ground-based automation will not allow the intruder to climb into LOS in this case, based on an implicit right-of-way rule. If this is a reliable rule, then AOP does not need to react to the climbing intruder in this case, even if it does not know the intruder's target altitude. However, there remains the risk of altitude overshoot by the intruder aircraft regardless of its clearance.

In the inverse of this case, the intruder would be descending from above the ownship, causing a conflict, although the intruder is planning to level out above the ownship. This case, although certainly possible, was not observed during the experiment.

\section{Cause 3: Intruder descends into the ownship.}

The intruder is flying level above the ownship and the pair are conflict free. Then, the intruder begins a descent, which causes a conflict with the ownship. (4 of 35 cases)

Example: (Conflict 10) The ownship is level at FL380 and the intruder is level at FL390, flying on similar tracks. The intruder beings a descent and AOP detects a conflict with nearly zero warning. AOP, unable to find a conflict resolution maneuver, instructs the pilot to maintain current track and altitude. The intruder does not pass closer than 6 nmi laterally to the ownship, and a LOS does not occur. The conflict clears once the intruder levels out at FL370. This conflict is shown in Fig. 3.

The mitigations for this case are similar to those previously discussed:

- AOP needs earlier information about the intruder's impending descent. If the intruder's planned TOD is broadcast as a trajectory change point, then AOP can react in advance and provide the pilot with more warning time.

- AOP must be overly conservative and assume any aircraft can descend at any time. Recognizing the inevitability that the intruder will need to descend, AOP could act preemptively and provide a route modification to the AFR pilot that increases the lateral separation between the two aircraft. 

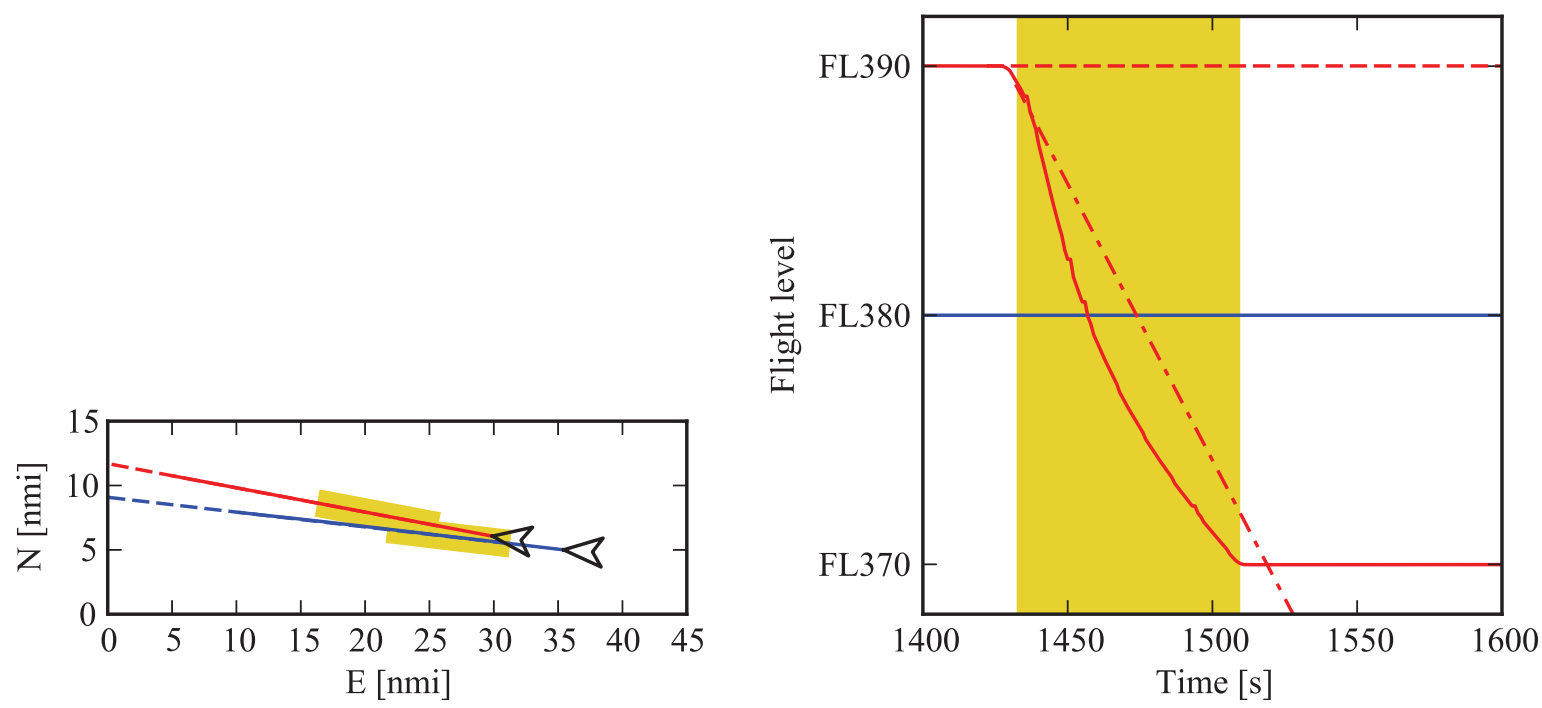

Figure 3. Conflict 10 trajectories. Left: lateral, right: vertical.

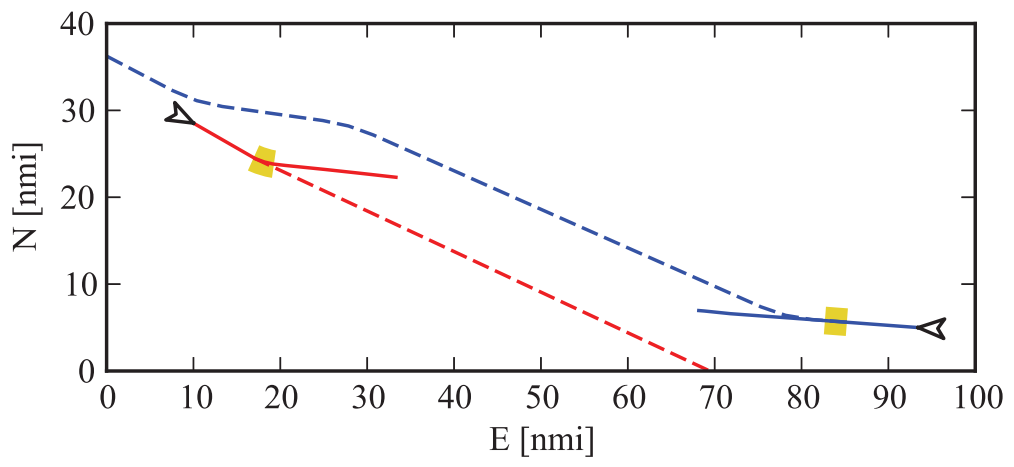

Figure 4. Conflict 19 lateral trajectories.

- The controller must vector the intruder to achieve lateral separation before clearing the intruder to descend. It is interesting in this case that the aircraft were laterally separated throughout the intruder's descent, but AOP detected the conflict due to its use of the 8 nmi IFR buffer.

\section{Cause 4: Intruder turns into the ownship.}

The intruder turns, creating a conflict with the ownship. (4 of 35 cases)

Example: (Conflict 19) The ownship and intruder are both level at FL370. The ownship has a planned right turn upcoming on the active route, which is conflict-free with the intruder. The intruder then makes a left turn, and AOP detects a conflict with 4:28 min of warning. AOP advises the pilot to remain on the current track and not to make the planned turn, thus resolving the conflict. This conflict is shown in Fig. 4.

This conflict was caused by the intruder's unannounced turn. The mitigations are similar to the previouslydiscussed vertical cases:

- AOP needs information about the intruder's turn. If the turn is a part of the intruder's flight plan or FMS active route, then it can be shared via an ADS-B trajectory change point or a data link from the ground. If the intruder is flying a vector from ATC, then the target track to which the intruder is turning can be shared via the ADS-B target state report.

- Without the trajectory information, AOP must be overly conservative, assuming any intruder can turn at any time 
and cause such a conflict. This requires a lateral buffer, such as the $8 \mathrm{nmi}$ IFR buffer employed here. Rather than simply increasing the separation distance, the buffer may be dependent on the speed and geometry of the ownship and intruder trajectories. This solution comes at the cost of increased airspace volume use and a loss of trajectory efficiency.

- The controller or ground-based automation must be restricted from issuing this heading change and creating this pop-up conflict. The controller should wait until the aircraft pass each other before clearing the intruder to turn, or alternatively clear the intruder to a different vector that will give the AFR aircraft sufficient warning time to resolve the conflict.

This cause was also observed with climbing intruders, where the intruder made a turn during a climb that caused the pop-up conflict.

\section{Cause 5: Transient intruder vertical speed creates a brief false alarm.}

When the intruder's vertical speed is changing, such as during top of climb or top of descent, AOP can detect a brief conflict even though the aircraft are actually conflict-free. (11 of 35 cases)

Example: (Conflict 2) The ownship is level at FL390 and the intruder is climbing from below on a crossing track from the left. The pair are conflict free while the intruder is climbing. However, at the moment the intruder levels out at FL380, AOP detects a conflict with 2:30 min until LOS. The ownship turns right to resolve the conflict even though the intruder is not a threat. This conflict is shown in Fig. 5.
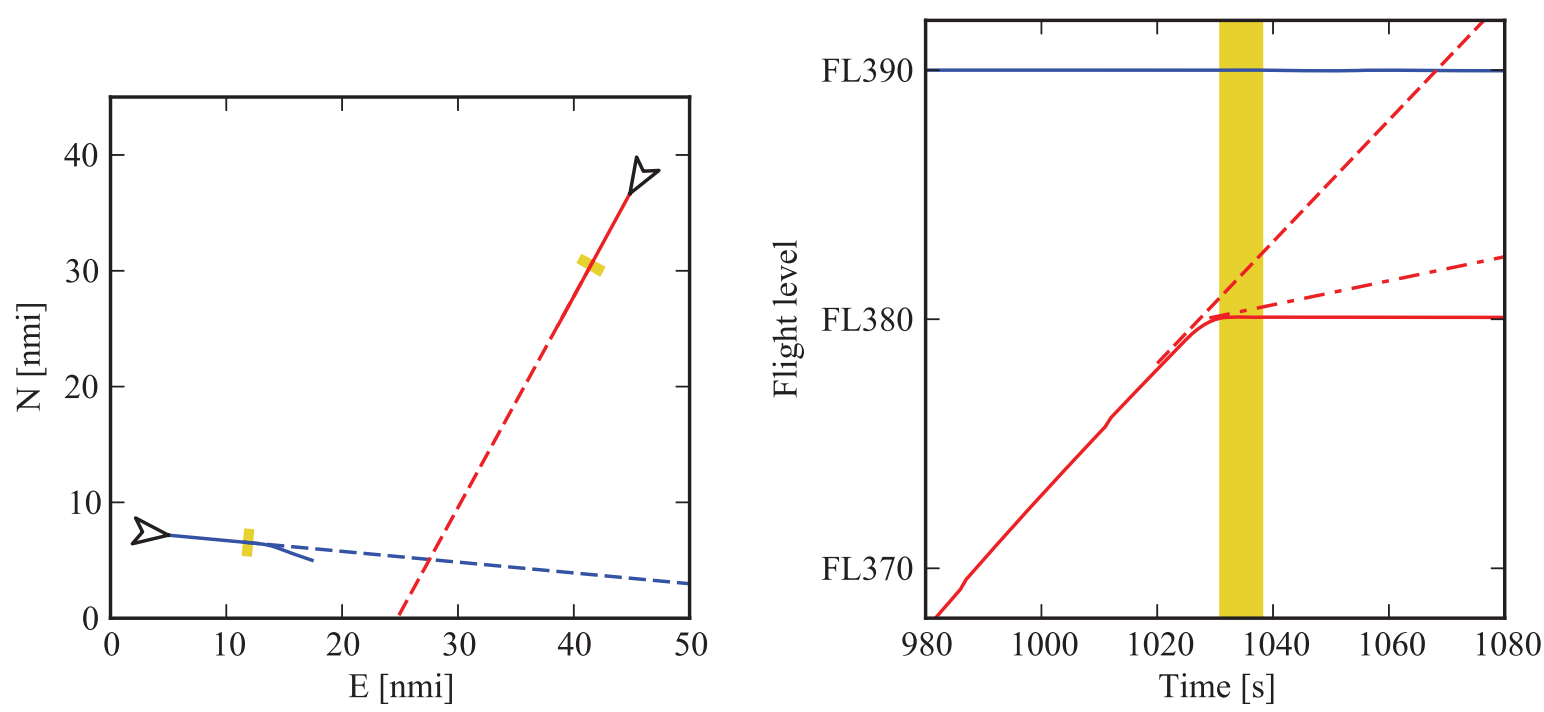

Figure 5. Conflict 2 trajectories. Left: lateral, right: vertical.

This is a spurious false alarm caused by the way AOP performs trajectory prediction based on the ADS-B state vector. As the intruder levels out, its vertical rate reduces from about 1500 feet per minute (fpm) to zero. However, during this vertical deceleration, at one instant the intruder broadcasts a state vector indicating a vertical speed of $290 \mathrm{fpm}$. At this instant, AOP creates a trajectory projection based on the instantaneous vertical speed and assumes the intruder will continue in a slow climb at this rate. AOP detects a conflict with the intruder based on this trajectory prediction, which lasts for about $10 \mathrm{~s}$.

There is no fault on the part of the controller in this case: the intruder was never a problem for the ownship, either during climb or during level off. Potential mitigations:

- Within AOP's current design, the problem is obviated by providing trajectory intent information, either via the target altitude or a vertical trajectory change point. With this information, AOP does not have to rely on the less-accurate state vector projection.

- AOP receives the ADS-B state vector from the traffic at a frequency of 1 hertz, and could potentially use the information about the intruder's changing vertical speed to better predict its future trajectory through mode identification and hybrid state estimation. ${ }^{6}$ 
In other examples, the transient detection occurred as the intruder began to descend from above the ownship, and the instantaneous vertical speed projection gave an initially shallower descent.

Cause 6: Improper climb procedure by the AFR pilot.

One of the conflicts was clearly caused by the AFR pilot not following proper procedure, not due to a maneuver by the IFR aircraft.

Example: (Conflict 3) The ownship is level at FL350 and the intruder (A) is level at FL360 approaching from the opposite direction, and the pair are conflict-free. The ownship detects a conflict with a different intruder (B) with 9:58 min of warning. The pilot selects a vertical resolution provided by AOP, which places a step climb to FL360 on the ownship's route in $42 \mathrm{nmi}$. However, rather than waiting to reach the step waypoint, the ownship pilot executes an immediate cruise altitude change to FL360, causing a pop-up with the intruder (A) with 3:42 of warning. The ownship executes a right turn during the climb in order to resolve the conflict. The pop-up with (A) is shown in Fig. 6.
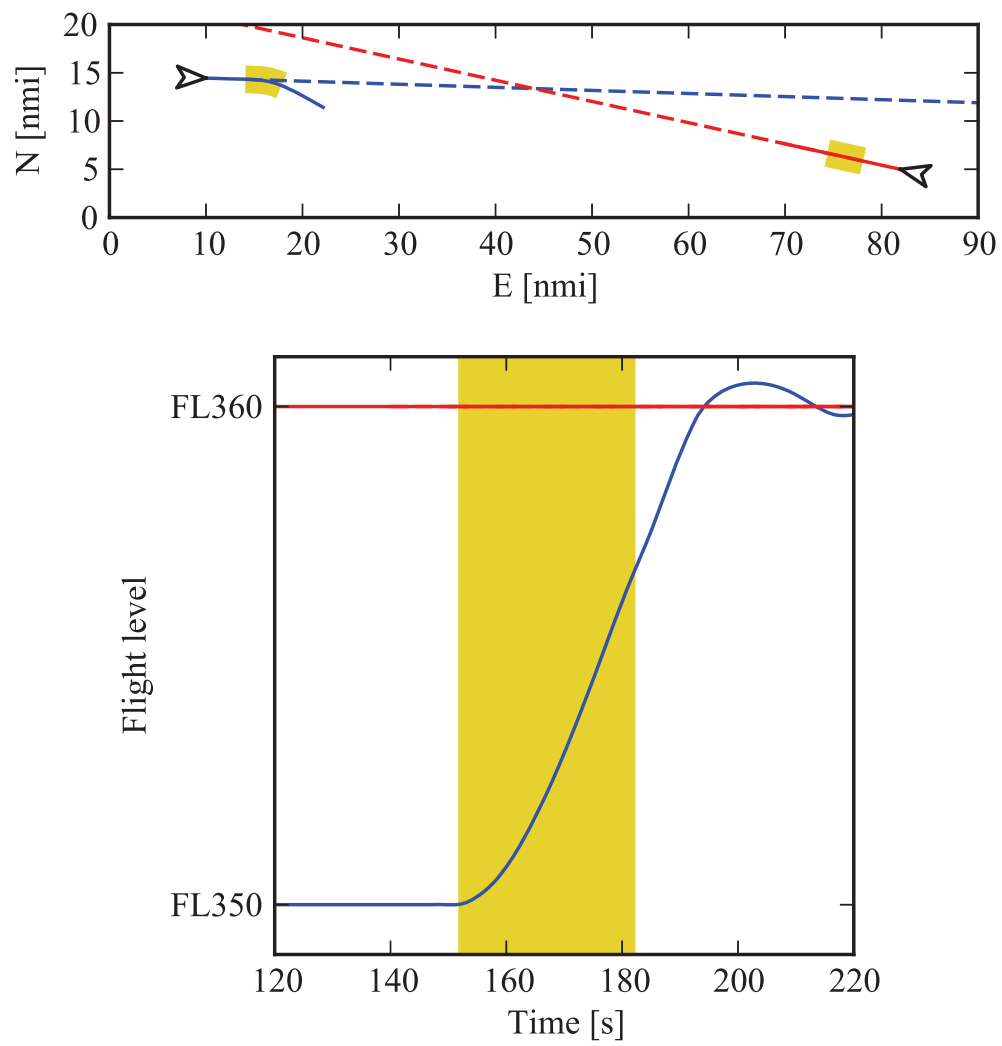

Figure 6. Conflict 3 trajectories. Top: lateral, bottom: vertical.

This conflict can be considered a blunder on the part of the AFR pilot. The best mitigation in this case is for the AFR pilot to follow the proper procedure for executing a vertical conflict resolution. The pilot should wait to climb at the step point rather than climbing immediately. Further, in the event that the pilot would like to climb sooner, AOP should be consulted to verify that the proposed trajectory change is conflict-free before executing. The pilot is not permitted to execute a maneuver that creates such a conflict according to the AFR rules and procedures. 


\section{Summary and Conclusions}

The AFR concept and AOP tool have been designed to i) enable the AFR pilot to take on responsibility for separation under en route conditions within acceptable workload limits; ii) to do so in IFR airspace alongside traffic of mixed equipage without substantially changing the air traffic control system as it exists today; and iii) in doing so provide immediate business-case benefits to operators that choose to equip. The pair of experiments described previously addressed goals i) and ii), and the Langley experiment presented here focused on i) specifically.

In the exploratory scenarios analyzed here, there were no losses of separation, and AFR/AOP was successful from this perspective. However, due to the nature of the near-term traffic environment, the AFR operation experienced a number of short-term, pop-up conflicts with IFR aircraft with less than 5 min of warning time for conflict resolution by the AFR pilot. Conflicts with much less than 5 min of warning were generally considered to be unacceptable to the pilots and controllers who participated in the experiment.

This paper explored the common causes of these conflicts, generally relating to the difficulty of predicting the trajectory of a climbing intruder who is not sharing any trajectory intent information. Three general approaches for mitigating these conflicts were identified:

1. It is difficult to avoid a maneuvering aircraft whose trajectory intent is not known. Beyond the mandated ADS-B state vector, AOP has been designed to incorporate information such as the target state report and trajectory change points into its conflict detection process. This experiment ${ }^{3}$ and previous work ${ }^{7}$ have shown the dramatic value of a small amount of near-term trajectory intent on the performance of AOP's conflict detection algorithms. Within AOP's current design, the availability of this information reduces the number of conflicts overall as well as the required amount of higher-workload tactical maneuvering.

This information could conceivably be shared via ADS-B in the future, although there is no strong business case for the IFR operator to equip to broadcast this information if it only benefits the AFR operation. Alternatively, the System Wide Information Management ${ }^{8}$ component of NextGen may provide a method of delivery for traffic trajectory information from the ground to the AFR flight deck.

2. Without accurate trajectory intent information, the algorithms within AOP must be much more conservative in the vicinity of IFR aircraft. This approach must take into account not only the uncertainty in the prediction of the intruder's nominal trajectory based on the current state vector, but also the uncertainty in the intruder's short-term intent. This can be accomplished through the use of intelligent, geometry-dependent airspace buffers in both the lateral and vertical dimensions that ensure a sufficient amount of warning time for the AFR aircraft to respond to any IFR conflict. The same problem can be posed as one of flexibility preservation ${ }^{9}$ or complexity avoidance: AOP should detect upcoming regions of high traffic density or other difficulty and preemptively offer an alternative route - one with fewer expected conflicts - to the pilot.

The principal feasibility question of this approach is: can such a buffer be implemented without consuming an undue amount of airspace and causing an impractical number of false alarms? If the required IFR buffer is too large, then the impact to the feasibility and benefits case for AFR could be detrimental.

3. Outside of AOP, other allocations of separation responsibility between the IFR controller and the AFR pilot warrant examination. As the automation support tools available to the controller become more sophisticated, they could be designed with the self-separating aircraft in mind. Additionally, a more equitable distribution of conflict priority in certain geometric encounters between IFR and AFR aircraft could make all the difference in the feasibility of the overall mixed operation. NASA is currently examining other such function allocations, outside the "IFR always has priority, AFR always gives way" approach presented here.

This project will likely move forward on all three of these fronts. It is clear that all three issues are interrelated, and design decisions about one cannot be made without considering the others.

This work is motivated in part by an important prior result: that many AFR aircraft can be introduced to IFR airspace without increasing the controller workload..$^{10}$ If the issues related to pop-up conflicts described in this paper can be solved within the paradigm of "AFR avoids IFR," then this concept has the potential to be introduced sooner than other concepts requiring more extensive deployment of advanced ground-based decision-support automation. This could reduce delays and increase capacity throughout the National Airspace System, and provide more optimal trajectories as an incentive for operators to equip. 


\section{Appendix: Conflict Data}

Table 2 lists the 35 conflicts presented in Section III. Each conflict is given an identifying ID. The amount of warning time and the ownship and intruder vertical conditions at initial condition are also listed. Finally, each conflict is associated with one of the conflict causes presented in Section III, with the exception seven cases marked by (-): these are primarily false alarms attributed to an AOP conflict detection issue that is beyond the scope of this paper.

Table 2. Enumerated pop-up conflicts.

\begin{tabular}{|c|c|c|c|c|}
\hline Conflict ID & Warning [s] & Ownship & Intruder & Cause ID \\
\hline 1 & 92 & LVL & TOC & 1 \\
\hline 2 & 150 & LVL & TOC & 5 \\
\hline 3 & 222 & BOC & LVL & 6 \\
\hline 4 & 117 & LVL & $\mathrm{BOC}$ & 2 \\
\hline 5 & 276 & LVL & CLB & 4 \\
\hline 6 & 289 & LVL & TOC & 5 \\
\hline 7 & 125 & TOD & BOD & 1 \\
\hline 8 & 12 & CLB & LVL & - \\
\hline 9 & 0 & LVL & TOD & 3 \\
\hline 10 & 0 & LVL & TOD & 3 \\
\hline 11 & 155 & LVL & TOD & 3 \\
\hline 12 & 48 & CLB & LVL & - \\
\hline 13 & 76 & LVL & TOC & 5 \\
\hline 14 & 203 & LVL & CLB & 4 \\
\hline 15 & 265 & LVL & TOC & 5 \\
\hline 16 & 273 & LVL & TOC & 5 \\
\hline 17 & 165 & LVL & TOD & 3 \\
\hline 18 & 221 & LVL & TOC & 1 \\
\hline 19 & 268 & LVL & LVL & 4 \\
\hline 20 & 209 & LVL & LVL & 4 \\
\hline 21 & 241 & $\mathrm{BOC}$ & CLB & - \\
\hline 22 & 16 & TOC & LVL & - \\
\hline 23 & 12 & TOC & LVL & - \\
\hline 24 & 198 & LVL & TOC & 5 \\
\hline 25 & 274 & LVL & TOC & 1 \\
\hline 26 & 121 & LVL & BOC & 2 \\
\hline 27 & 177 & LVL & TOC & 5 \\
\hline 28 & 55 & LVL & BOC & 2 \\
\hline 29 & 281 & LVL & TOC & 5 \\
\hline 30 & 13 & TOC & LVL & - \\
\hline 31 & 282 & LVL & TOC & 5 \\
\hline 32 & 247 & LVL & BOC & 2 \\
\hline 33 & 18 & BOC & LVL & - \\
\hline 34 & 228 & LVL & TOC & 5 \\
\hline 35 & 233 & LVL & TOC & 5 \\
\hline
\end{tabular}

\section{Acknowledgments}

The author would like to thank colleagues Sally Johnson and David Wing for their support and advice during the analysis and writing of this paper. 


\section{References}

${ }^{1}$ Wing, D. J. and Cotton, W. B., Autonomous Flight Rules: A Concept for Self Separation in US Domestic Airspace, NASA/TP-2011-217174, 2011.

${ }^{2}$ Wing, D. J. and Cotton, W. B., "For Spacious Skies: Self-Separation with 'Autonomous Flight Rules' in US Domestic Airspace," AIAA2011-6865, September 2011.

${ }^{3}$ Wing, D., Prevot, T., Lewis, T., Cabrall, C., Johnson, S., Homola, J., et al., "Pilot and Controller Evaluations of Separation Function Allocation in Air Traffic Management," Tenth USA/Europe Air Traffic Management R\&D Seminar, Chicago, IL, June 2013.

${ }^{4}$ Department of Transportation, Federal Aviation Administration, Automatic Dependent Surveillance-Broadcast (ADS-B) Out Performance Requirements To Support Air Traffic Control (ATC) Service; Final Rule, 14 CFR Part 91, May 2010.

${ }^{5}$ Karr, D. A., Vivona, R. A., Roscoe, D. A., DePascale, S. M., and Wing, D. J., "Autonomous Operations Planner: A Flexible Platform for Research in Flight-Deck Support for Airborne Self-Separation," AIAA-2012-5417, September 2012.

${ }^{6}$ Hwang, I. and Seah, C. E., "Intent-Based Probabilistic Conflict Detection for the Next Generation Air Transportation System," Proceedings of the IEEE, Vol. 96, No. 12, December 2008

${ }^{7}$ Lewis, T. A., Phojanamongkolkij, N., and Wing, D. J., "The Effects of Limited Intent Information Availability on Self-Separation in Mixed Operations," 2012 Integrated Communications Navigation and Surveillance Conference, Herndon, VA, April 2012.

${ }^{8}$ Federal Aviation Administration, System Wide Information Management (SWIM) Final Program Requirements, Segment 1, May 2007.

${ }^{9}$ Idris, H., Delahaye, D., and Wing, D., "Distributed Trajectory Flexibility Preservation for Traffic Complexity Mitigation," Eighth USA/Europe Air Traffic Management R\&D Seminar, Napa, CA, June 2009.

${ }^{10}$ Barhydt, R., Kopardekar, P., Battiste, V., Doble, N., Johnson, W., Lee, P., et al., "Joint NASA Ames/Langley Experiment Evaluation of Integrated Air/Ground Operations for En Route Free Maneuvering," Sixth USA/Europe Air Traffic Management R\&D Seminar, Baltimore, MD, June 2005 